https://helda.helsinki.fi

\title{
MDL-based Models for Transliteration Generation
}

\section{Nouri, Javad}

2013

Nouri , J , Pivovarova , L \& Yangarber , R 2013 , MDL-based Models for Transliteration Generation . in Unknown host publication . SLSP 2013: International Conference on Statistical Language and Speech Processing , Tarragona, Spain , 29/07/2013 . < http://grammars.grlmc.com/slsp2013/ >

http://hdl.handle.net/10138/42904

publishedVersion

Downloaded from Helda, University of Helsinki institutional repository.

This is an electronic reprint of the original article.

This reprint may differ from the original in pagination and typographic detail.

Please cite the original version. 


\title{
MDL-based Models for Transliteration Generation
}

\author{
Javad Nouri, Lidia Pivovarova, and Roman Yangarber \\ University of Helsinki, Finland
}

\begin{abstract}
This paper presents models for automatic transliteration of proper names between languages that use different alphabets. The models are an extension of our work on automatic discovery of patterns of etymological sound change, based on the Minimum Description Length Principle. The models for pairwise alignment are extended with algorithms for prediction that produce transliterated names. We present results on 13 parallel corpora for 7 languages, including English, Russian, and Farsi, extracted from Wikipedia headlines. The transliteration corpora are released for public use. The models achieve up to $88 \%$ on word-level accuracy and up to $99 \%$ on symbol-level F-score. We discuss the results from several perspectives, and analyze how corpus size, the language pair, the type of names (persons, locations), and noise in the data affect the performance.
\end{abstract}

\section{Introduction}

The task of machine transliteration involves mapping the representation of a word to another language, typically using a different alphabet, based on its sound or spelling, rather than its meaning. Transliteration is commonly applied to proper names, as well as to terms in rapidly growing areas, such as medicine or technology, [12]. Two principal applications for machine transliteration are machine translation and information search-multilingual information retrieval (IR), information extraction (IE), and named-entity recognition (NER). While in machine translation the goal may be to produce only one correct transliteration for each word, in other tasks we may wish to produce several possible transliterations, and merge different variants of the same name.

There are two main approaches to machine transliteration: transliteration generation and transliteration mining (discovery). In transliteration generation one builds a transliteration model, which takes a source named entity as input and produces its representations in the target language as output.

Transliteration generation can be considered in a broad sense as a special case of alignment and transduction of words. The similarity of machine transliteration and alignment of etymologically-related words have been observed by [15], who applied the same model, a finite state transducer, for both tasks. In [1], transliteration is mentioned in the broader context of linguistic string-transduction tasks, such as paraphrasing, morphological transformation and co-reference resolution. 
The definition that [3] proposes for cognate - "words with a common form and meaning across the languages" - is applicable to both transliterated and etymologically related words. Machine transliteration is, of course, a different task from cognate alignment. The main principle for word changes in etymology is the regularity of sound change, whereas transliteration of names may not always follow regular rules. Transliteration does not always follow pronunciation in practice - it can be based on script, or tradition, or on translation. For exam-

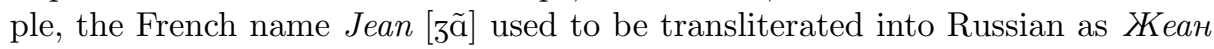
[zean], i.e., based on its French spelling rather than its pronunciation. The same name may be transliterated into another language differently depending on tradition: e.g., the name of the famous Russian author Лев Толстой is commonly translated into English as Leo Tolstoy; the name of his son, which is identical in Russian, is commonly transliterated as Lev Tolstoy. Furthermore, transliteration rules may be different in different domains [6].

Thus, there may be more noise in transliteration data than in etymological data. On the other hand, words in remotely related languages, (e.g., FinnishHungarian), will have substantially more complex correspondences. Therefore, while the tasks of transliteration and etymological alignment may be similar from the algorithmic point of view, it is not a priori obvious that the same algorithms will work for both tasks.

The models that we use for transliteration were originally developed for automatic discovery of patterns in etymological sound change, [17]. We apply these models to 13 parallel corpora in 7 languages: Farsi, English, Russian, Greek, Hebrew, French, and Japanese (Katakana script for foreign names), which are extracted from Wikipedia headlines using language links. We report results on automatic transliteration of names of American actors from English into four languages that use different kinds of writing systems: alphabetic (Russian and Greek) and consonantal (Farsi and Hebrew). We also examine the performance on data of different semantic type - person names vs. location names. For example, for English-Russian transliteration we use 3 different datasets: person names of English origin (American actors), person names of Russian origin (Russian writers), and location names of Russian origin (Russian cities).

As far as we are aware, the comparison of transliteration results among different semantic types has not been addressed in the literature to date; [10] studied the influence of the origin of names and of noise in the data on the results of transliteration, but they did not apply their method to words of different types.

\section{Related work}

A comprehensive survey in [9] classifies machine transliteration generation methods into several broad categories: rule-based, phonetics-based, spelling-based, hybrid - a mix of spelling and phonetics, and combined - applying several methods and then selecting the best transliteration via re-ranking. According to this classification scheme, the models we use would be classified as spelling-based, as they use only the spelling of the words with no information about their pronun- 
ciation. Some of our etymological models use phonetic features [19]; they will be applied to the transliteration task in future work.

The most common approach for spelling-based transliteration uses sourcechannel models, $[11,4]$. Many methods used for machine transliteration are adopted from phrase-based statistical machine translation $[16,5]$. The importance of the representation of word-pairs is emphasized in [6], i.e., the features used in the transliteration task. The representation in [6] combines uni-grams and bi-grams, which are then used as features in an optimization task. It was shown in [12] that sequential n-grams outperform the bag-of-word n-gram models.

A Minimum Description Length (MDL) approach to transliteration is described in [22]. This work, as well as others [13,8], uses the Expectation-Maximization (EM) algorithm: in the Expectation stage the probabilities of substring correspondence are counted; in the Maximization stage word pairs are re-aligned using these probabilities.

Automatic transliteration and evaluation has been explored in a series of workshops on Named Entities, which organized shared tasks in transliteration generation and mining [24].

\section{Data}

We use datasets that we extracted from cross-language links in the Wikipedia. We did not try to extract as much data as possible; rather, we focused on language and on topical homogeneity of each dataset. In this paper, we present work on Wikipedia dumps dated up to 12 December $2012 .{ }^{1}$ We used only the page titles, language links, and category links. The full text of the articles is not used in the transliteration task, with one exception, below. To extract the datasets we used Wikipedia Categories; we tried to focus on categories that can guarantee higher homogeneity in the data. For example, the majority of names in category American actors are of English origin, and most of the names in category Russian Writers are of Russian origin. However, there are many exceptions among person names; this is especially true for languages such as English or Russian, which are in use across wide geographic areas.

We also used location names, since toponyms may be more stable and more consistent than person names. For the dataset of Iranian Locations, we parsed the content of the corresponding Wikipedia pages. Since the number of titles for Iranian cities in the Russian Wikipedia was small, we collected names of towns and locations from Russian-Wikipedia pages about Iranian Shahrestans (counties). The data used our experiments is summarized in Table 1.

Each dataset was semi-automatically cleaned. Some amount of noise was intentionally left in data; e.g., the name of the Russian city Санкт-Петербург ([Sankt-Peterburg]) is usually "transcribed" into English as Saint-Petersburg, because this is a commonly accepted translation, although it is not phonetically accurate. We removed patronymics, which are very common in the Russian data,

\footnotetext{
${ }^{1}$ Wikipedia dumps are available under the GNU Free Documentation License and Creative Commons License at the Wikimedia Web-site.
} 


\begin{tabular}{|c|c|c|c|c|c|}
\hline Dataset & $\begin{array}{c}\text { Language } \\
\text { pair }\end{array}$ & $\begin{array}{l}\text { Size: \# } \\
\text { of pairs }\end{array}$ & Dataset & $\begin{array}{c}\text { Language } \\
\text { pair }\end{array}$ & $\begin{array}{l}\text { Size: \# } \\
\text { of pairs }\end{array}$ \\
\hline \multirow[t]{4}{*}{ American Actors } & En-Ru & 1471 & \multirow[t]{4}{*}{ Russian Cities } & $\mathrm{Ru}-\mathrm{En}$ & 1136 \\
\hline & $\mathrm{En}-\mathrm{He}$ & 1245 & & $\mathrm{Ru}-\mathrm{Fa}$ & 870 \\
\hline & $\mathrm{En}-\mathrm{Fa}$ & 840 & & $\mathrm{Ru}-\mathrm{Fr}$ & 828 \\
\hline & En-Gr & 407 & & $\mathrm{Ru}-\mathrm{Jp}$ & 317 \\
\hline Russian Writes & $\mathrm{Ru}-\mathrm{En}$ & 1462 & French Cities & Fr-Ru & 828 \\
\hline Iranian Cities & $\begin{array}{l}\text { Fa-En } \\
\text { Fa-Ru }\end{array}$ & $\begin{array}{l}439 \\
469\end{array}$ & Iranian Locations & $\mathrm{Fa}-\mathrm{Ru}$ & 1893 \\
\hline
\end{tabular}

Table 1: Transliteration corpora/datasets, extracted from Wikipedia headlines and language links. The language codes are: En-English, Fa-Farsi, Fr-French, Gr-Greek, He-Hebrew, Jp-Japanese (Katakana), Ru-Russian.

since in most cases they are omitted in other languages. We also removed all accent marks from the Greek dataset. Since these marks are obligatory in Greek script, transliteration models based on these data cannot be used in real-world application, though this data still interesting for a transliteration task. ${ }^{2}$

It was stated in [24] that "a reasonably large" dataset for the transliteration task should consist of $\sim 10000$ name pairs, which is orders of magnitude larger than our datasets. Some authors report satisfactory results using considerably smaller datasets. For example, a word-level accuracy of $33 \%$ for Arabicto-English transliteration is reported using a training set of only 935 name pairs, and an accuracy of $46 \%$ for Russian-to-English, using a training set of 545 name pairs [23]. In this paper we present models that achieve good results (Section 6) on relatively small datasets; DirecTL+ [7], which we use as a baseline for comparison, also demonstrates reasonable results on our datasets.

\section{Method}

We use Etymon, a set of MDL-based models that we developed for analyzing etymological data, as the basis for our transliteration models. ${ }^{3}$ The collection of models is described in $[17,19,18]$. The models take as input a phonetic representation of genetically related words, or cognates, and aim to discover regular phonetic changes between languages within a language family. This is done by searching for the best pairwise alignment of words, by optimizing the description length of the alignments. Transliteration is an analogous task, since in order to learn how to transliterate from one language to another, it seems that a natural prerequisite is to align the words in the training set. Thus, it seemed reasonable to suppose that we could use these models for aligning the data. After alignment, we introduce a set of prediction procedures for performing the actual transliteration-based on the alignment. We briefly describe the models; detailed explanations can be found in $[17,19]$ — and the prediction procedure.

\footnotetext{
${ }^{2}$ We consider recovery of accents a separate task, beyond the scope of this work.

3 The tools are publicly available from http://etymon.cs.helsinki.fi/
} 


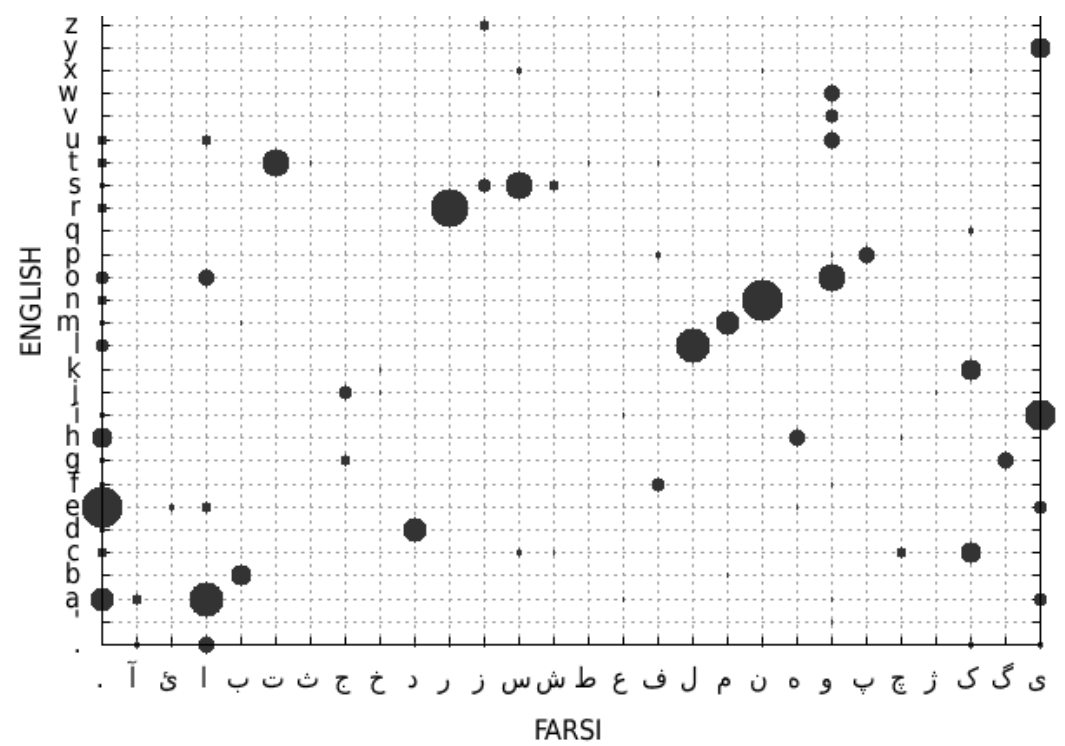

Fig. 1: English-Farsi alignment matrix; American Actors dataset. The size of each ball indicates the probability of the corresponding symbol-pair alignment.

\section{$4.11 \times 1$ Alignment}

Our baseline $1 \times 1$ model finds an optimal alignment, where each symbol of the source word may align to at most one symbol of the target word, with possible insertions or deletions. Information about the context of the symbols is not used.

For example, the alignment matrix for English to Farsi transliteration on the American Actors is shown in Figure 1. The matrix shows that, e.g., English e is most frequently aligned to "." due to omission of short vowels in Farsi script. Mapping English a to Farsi $T$ is rare, as seen from the size of the corresponding bubble; this happens when a designates [a] that is situated at the beginning of the word. The $1 \times 1$ model is unable to capture this rule; the only information it uses is that $\mathbf{a}$ is transliterated to I more frequently than to any other symbol.

\section{$4.22 \times 2$ Alignment}

The $2 \times 2$ model extends the $1 \times 1$ model, by allowing for alignment of up to two consecutive symbols at a time on each level; this model also takes into account the start and end word boundaries. Unlike the $1 \times 1$ model, this model captures some information about the symbols' context when learning the correspondences. For example, it should discover cases where one symbol in language $A$ corresponds to two symbols in language $B$; e.g., Russian ' $\mathrm{'}$ ' is often transliterated as "ch" in English - the $1 \times 1$ model is by definition unable to discover such correspondences. The $2 \times 2$ model can also discover that certain symbols are transliterated 
differently when they appear at the beginning or at the end of a word, as in the example of a and T mentioned above, in Section 4.1. For example, the name Alda from the American Actors dataset is correctly transliterated into Farsi as آلدا by the the $2 \times 2$ model — with $T$ at the beginning. The $1 \times 1$ model incorrectly predicts the transliteration الدا -with I as the most probable correspondence for $\mathbf{a}$ in both initial and final position, unable to exploit the context information.

\subsection{Prediction}

Once all word pairs in the training set are aligned, the discovered symbol correspondences can be used to predict transliterations for new, unseen words. The result of the convergence of the alignment algorithm is a count matrix, such as one shown in Figure 1, which indicates how often each symbol (in the $1 \times 1$ model) — or pair of symbols (in the $2 \times 2$ model) — of the source language is aligned to symbols in the target language in an optimal way. We have implemented an algorithm that predicts the target representation of a given source word based on the alignment. Theoretically, this is done by searching among all possible strings in the target language to select the string that yields the lowest cost under the model, when aligned with the source word. In practice, this can be achieved efficiently, by using simple table lookup for the baseline $1 \times 1$ model, and by a Dynamic Programming algorithm for the more complex models. Prediction based on the $1 \times 1$ model is straightforward, since symbols are aligned independently of their context; we assign to each source symbol the single target symbol to which it is cheapest to align:

$$
t_{i}=\arg \min _{t \in T} L\left(s_{i}, t\right)
$$

where $s_{i}$ is the $i$ th symbol in source word, $T$ is the alphabet of the target language augmented with the special symbol '.' to allow for deletions, and $L(x, y)$ is the cost (code-length) of aligning the source-language symbol $x$ to the targetlanguage symbol $y$ under the learned model.

The $2 \times 2$ prediction is more complicated, since it is possible to align zero, one, or two source symbols to symbols of the target language, and we need to choose the lowest cost alignment for the entire source word. We solve this optimization problem using Dynamic Programming (DP). To predict a target word, the algorithm starts from the beginning of the source word, and for each symbol $s_{i}$, finds the best prediction up to $s_{i}$ based on previously computed partial alignments. The algorithm computes the cost $L(i)$ of the best prediction up to $s_{i}$, for all $i$. Thus, for predicting the best target sequence corresponding to the source word up to the $i$-th symbol, $s_{i}$, the possible final candidate alignments are in the set $C$, where:

$$
C=\left\{\left(s_{i}: .\right),\left(s_{i}: t\right),\left(s_{i}: t t^{\prime}\right),\left(s_{i-1} s_{i}: .\right),\left(s_{i-1} s_{i}: t\right),\left(s_{i-1} s_{i}: t t^{\prime}\right)\right\},
$$

and $t$ and $t^{\prime}$ are symbols from the target alphabet. Each of these 6 alignments has a fixed cost under the learned $2 \times 2$ model. The optimal prediction up to the $i$-th source symbol is given by minimizing the sum of one of these alignments, plus the cost $L(i-1)$ of the optimal alignment up to symbol $s_{i-1}$, for the first 
three candidates in $C$, or $L(i-2)$, the optimal cost up to $s_{i-2}$ for the last three candidates in $C$,- where $L(i-1)$ and $L(i-2)$ have been pre-computed by DP previously. Using this approach the best target word - under the model - can be predicted in linear time.

\section{Evaluation}

The quality of machine transliteration depends on the ultimate task for which transliteration is being developed. In multilingual IE and IR, the system may make use of multiple possible variants. For example, in many cases more than one transliteration may be acceptable for a particular name, but if the system populates a database with events or relationships, identifying and merging different references to the same real-world entity is needed across multiple sources, $[21,20]$. In a multi-lingual setting, this capability is indispensable, $[2,14]$.

Here, we evaluate performance of the transliteration models at the word level and at the symbol level. The most common word-level measure is accuracy [9]:

$$
A=\frac{\text { number of correct transliterations }}{\text { total number of test words }}
$$

Symbol-based evaluation measures are more diverse than word-based ones; in general, they are based on an edit distance between the system response and the expected transliteration. In this paper we use Normalized Edit Distance and Mean F-score. The normalized edit distance is computed as:

$$
N E D=\frac{\sum_{i} E D\left(c_{i}, r_{i}\right)}{\sum_{i}\left|c_{i}\right|}
$$

where $c_{i}$ is the expected transliteration for word $i, r_{i}$ is the system response, and $E D\left(c_{i}, r_{i}\right)$ is an edit distance (here, the Levenshtein edit distance).

The symbol-level Mean F-score [24] is based on the Longest Common Subsequence between an expected transliteration $c$ and the system response $r^{4}$ :

$$
L C S(c, r)=\frac{1}{2}(|c|+|r|-E D(c, r))
$$

Recall, Precision and F-score for a particular word are calculated on the basis of $L C S$ :

$$
R=\frac{L C S(c, r)}{|r|} \quad P=\frac{L C S(c, r)}{|c|} \quad F=2 \frac{R \times P}{R+P}
$$

We average the F-score over all words to get the mean over the entire data set.

Alongside our models, we use two other models for comparison. One is a naive baseline, where each symbol of source alphabet is transliterated as fixed symbol (or a string of symbols) from the target alphabet. In many cases this is a one-toone mapping, but there are many exceptions; e.g., the Russian щ corresponds to English shch while Russian ь is most frequently omitted in transliteration. We

${ }^{4}$ We slightly simplify all formulae here, assuming only one expected transliteration and one system response for each word. 
did not apply this baseline to Katakana, since it is difficult to make reasonable correspondence between Katakana and Russian symbols.

The second model we used for comparison is the open-source system DirecTL+ [7]. It uses aligned data as input for the training; for alignment, we use the M2M-aligner, an open source program by the same authors, $[8] .^{5}$

We evaluate the models' performance via leave-one-out cross-validation.

\section{Results}

The results are shown in Tables 2 and 3 , followed by the overall scores, average over all datasets that we tested. Although on some of the datasets, DirecTL+ beats the Etymon models, Etymon's performance appears higher overall.

One shortcoming of this evaluation scheme may be that only one correct answer is permitted for each word pair. For example, in the American Actors dataset, the English surname Murray is transliterated into Russian in two different ways: twice as Мюррей and twice as Мюррэй-therefore, for this name (Murray) any model can get at most 50\% accuracy at the word level. We did not measure how this ambiguity ultimately affects the evaluation results, though it is common for person names.

By comparison, location names are more consistent; in most cases the toponyms are older and represent a more homogeneous transliteration scheme. Loan words and repetitions are more rare among location names. Thus, the results on location data are in general higher. For example, if we consider the results on three English-Russian datasets, namely American Actors, Russian Writers and Russian Cities, we can see from the tables that for both forward and backward transliteration the highest performance is achieved on the Russian Cities dataset. Comparing the datasets Russian Writers and Russian Cities is informative: both datasets use the same language pair, have the same language of names origin, and approximately the same size. However, we observe a difference of $20 \%$ in word-level accuracy on Ru-En transliteration and $14 \%$ on En-Ru, due to differences in the nature of the names.

It is also interesting to compare the Iranian Cities and Iranian Locations datasets for Russian-Farsi transliteration. As was described in Section 3, the latter contains a list of the Shahrestan's locations with population over 800 . The dataset is four times larger, but it is also more noisy: Wikipedia editors seem to pay less attention to transliteration of smaller place names. In fact, we have found many inaccuracies among the Iranian Locations. For example, the Iranian place-name بوئين/buin/ appears in Russian as By /bu/. Due to such noise in the data, for these datasets we achieved approximately the same results according to all measures, although the number of word pairs in the Iranian Cities dataset (439) is four times smaller than in the Iranian Locations dataset (1893). This

\footnotetext{
${ }^{5}$ We use default parameters for both programs. It may be possible to achieve better results through elaborate tuning of the parameters, though we did not explore parameter tuning. By comparison, our Etymon models have no parameters to tune.
} 


\begin{tabular}{|c|c|c|c|c|c|c|c|}
\hline $\begin{array}{l}\text { Size: \# } \\
\text { of pairs }\end{array}$ & Model & \begin{tabular}{|c|} 
Word level \\
Accuracy
\end{tabular} & NED & \begin{tabular}{|c|} 
Mean \\
F-Score \\
\end{tabular} & $\begin{array}{c}\text { Word level } \\
\text { Accuracy }\end{array}$ & NED & \begin{tabular}{|c|} 
Mean \\
F-Score \\
\end{tabular} \\
\hline \multicolumn{8}{|c|}{ American Actors } \\
\hline \multirow{5}{*}{840} & & \multicolumn{3}{|c|}{$\mathrm{En} \rightarrow \mathrm{Fa}$} & \multicolumn{3}{|c|}{$\mathrm{Fa} \rightarrow \mathrm{En}$} \\
\hline & $1 \mathrm{x} 1$ & 0.223 & 0.256 & 0.866 & 0.081 & 0.371 & 0.785 \\
\hline & $2 \times 2$ & 0.393 & 0.180 & 0.904 & 0.080 & 0.346 & 0.805 \\
\hline & Baseline & 0.233 & 0.273 & 0.863 & 0.032 & 0.433 & 0.759 \\
\hline & DirecTL+ & 0.157 & 0.363 & 0.831 & 0.118 & 0.324 & 0.828 \\
\hline \multirow{5}{*}{407} & & \multicolumn{3}{|c|}{$\mathrm{En} \rightarrow \mathrm{Gr}$} & \multicolumn{3}{|c|}{$\mathrm{Gr} \rightarrow \mathrm{En}$} \\
\hline & $1 \mathrm{x} 1$ & 0.157 & 0.312 & 0.835 & 0.079 & 0.385 & 0.791 \\
\hline & $2 \mathrm{x} 2$ & 0.437 & 0.171 & 0.914 & 0.268 & 0.238 & 0.869 \\
\hline & Baseline & 0.179 & 0.343 & 0.833 & 0.101 & 0.456 & 0.767 \\
\hline & DirecTL+ & 0.342 & 0.232 & 0.887 & 0.140 & 0.417 & 0.794 \\
\hline \multirow{5}{*}{1245} & & \multicolumn{3}{|c|}{$\mathrm{En} \rightarrow \mathrm{He}$} & \multicolumn{3}{|c|}{$\mathrm{He} \rightarrow$ En } \\
\hline & $1 \mathrm{x} 1$ & 0.160 & 0.301 & 0.830 & 0.070 & 0.382 & 0.788 \\
\hline & $2 \times 2$ & 0.415 & 0.186 & 0.901 & 0.104 & 0.337 & 0.816 \\
\hline & Baseline & 0.074 & 0.426 & 0.803 & 0.043 & 0.430 & 0.762 \\
\hline & DirecTL+ & 0.160 & 0.331 & 0.850 & 0.131 & 0.327 & 0.832 \\
\hline \multirow{5}{*}{1471} & & \multicolumn{3}{|c|}{$\mathrm{En} \rightarrow \mathrm{Ru}$} & \multicolumn{3}{|c|}{$\mathrm{Ru} \rightarrow \mathrm{En}$} \\
\hline & $1 \mathrm{x} 1$ & 0.338 & 0.222 & 0.881 & 0.309 & 0.223 & 0.878 \\
\hline & $2 \times 2$ & 0.430 & 0.176 & 0.905 & 0.388 & 0.177 & 0.901 \\
\hline & Baseline & 0.298 & 0.25 & 0.871 & 0.282 & 0.250 & 0.868 \\
\hline & DirecTL+ & 0.387 & 0.214 & 0.890 & 0.373 & 0.189 & 0.901 \\
\hline \multicolumn{8}{|c|}{ Russian Cities } \\
\hline \multirow{5}{*}{1136} & & \multicolumn{3}{|c|}{$\mathrm{En} \rightarrow \mathrm{Ru}$} & \multicolumn{3}{|c|}{$\mathrm{Ru} \rightarrow \mathrm{En}$} \\
\hline & $1 \mathrm{x} 1$ & 0.448 & 0.113 & 0.936 & 0.509 & 0.082 & 0.957 \\
\hline & $2 \times 2$ & 0.762 & 0.040 & 0.978 & 0.881 & 0.018 & 0.99 \\
\hline & Baseline & 0.379 & 0.176 & 0.911 & 0.823 & 0.028 & 0.986 \\
\hline & DirecTL+ & 0.501 & 0.163 & 0.919 & 0.813 & 0.028 & 0.985 \\
\hline \multirow{5}{*}{870} & & \multicolumn{3}{|c|}{$\mathrm{Fa} \rightarrow \mathrm{Ru}$} & \multicolumn{3}{|c|}{$\mathrm{Ru} \rightarrow \mathrm{Fa}$} \\
\hline & $1 \mathrm{x} 1$ & 0.180 & 0.230 & 0.877 & 0.441 & 0.110 & 0.941 \\
\hline & $2 \times 2$ & 0.302 & 0.170 & 0.908 & 0.684 & 0.06 & 0.971 \\
\hline & Baseline & 0.125 & 0.264 & 0.861 & 0.507 & 0.098 & 0.949 \\
\hline & DirecTL+ & 0.325 & 0.190 & 0.900 & 0.514 & 0.100 & 0.952 \\
\hline \multirow{4}{*}{317} & & \multicolumn{3}{|c|}{$\mathrm{Ru} \rightarrow \mathrm{Jp}$} & \multicolumn{3}{|c|}{$\mathrm{Jp} \rightarrow \mathrm{Ru}$} \\
\hline & $1 \mathrm{x} 1$ & 0.013 & 0.552 & 0.695 & 0.016 & 0.377 & 0.779 \\
\hline & $2 \mathrm{x} 2$ & 0.565 & 0.126 & 0.935 & 0.3 & 0.145 & 0.922 \\
\hline & DirecTL+ & 0.022 & 0.742 & 0.687 & 0.287 & 0.159 & 0.915 \\
\hline \multirow{5}{*}{828} & & $\mathrm{Ru}$ & $\rightarrow \mathrm{Fr}$ & & $\mathrm{Fr}$ & $\rightarrow \mathrm{Ru}$ & \\
\hline & $1 \mathrm{x} 1$ & 0.389 & 0.124 & 0.933 & 0.355 & 0.154 & 0.919 \\
\hline & $2 \times 2$ & 0.697 & 0.051 & 0.973 & 0.668 & 0.065 & 0.965 \\
\hline & Baseline & 0.383 & 0.122 & 0.937 & 0.307 & 0.210 & 0.897 \\
\hline & DirecTL+ & 0.736 & 0.042 & 0.978 & 0.396 & 0.189 & 0.907 \\
\hline
\end{tabular}

Table 2: Transliteration results 


\begin{tabular}{|c|c|c|c|c|c|c|c|}
\hline $\begin{array}{l}\text { Size: \# } \\
\text { of pairs }\end{array}$ & Model & \begin{tabular}{|c|} 
Word level \\
Accuracy
\end{tabular} & NED & $\begin{array}{c}\text { Mean } \\
\text { F-Score }\end{array}$ & \begin{tabular}{|c|} 
Word level \\
Accuracy
\end{tabular} & NED & \begin{tabular}{|c|} 
Mean \\
F-Score \\
\end{tabular} \\
\hline \multicolumn{8}{|c|}{ Russian Writers } \\
\hline \multirow{5}{*}{1462} & & \multicolumn{3}{|c|}{$\mathrm{En} \rightarrow \mathrm{Ru}$} & \multicolumn{3}{|c|}{$\mathrm{Ru} \rightarrow \mathrm{En}$} \\
\hline & $1 \mathrm{x} 1$ & 0.400 & 0.153 & 0.920 & 0.415 & 0.126 & 0.936 \\
\hline & $2 \times 2$ & 0.634 & 0.091 & 0.953 & 0.689 & 0.073 & 0.962 \\
\hline & Baseline & 0.347 & 0.201 & 0.903 & 0.651 & 0.075 & 0.961 \\
\hline & DirecTL+ & 0.462 & 0.176 & 0.916 & 0.588 & 0.090 & 0.953 \\
\hline \multicolumn{8}{|c|}{ French Cities } \\
\hline \multirow{5}{*}{828} & & \multicolumn{3}{|c|}{$\mathrm{Ru} \rightarrow \mathrm{Fr}$} & \multicolumn{3}{|c|}{$\mathrm{Fr} \rightarrow \mathrm{Ru}$} \\
\hline & $1 \mathrm{x} 1$ & 0.088 & 0.338 & 0.806 & 0.113 & 0.357 & 0.805 \\
\hline & $2 \times 2$ & 0.148 & 0.297 & 0.835 & 0.381 & 0.182 & 0.905 \\
\hline & Baseline & 0.075 & 0.376 & 0.790 & 0.081 & 0.471 & 0.787 \\
\hline & DirecTL+ & 0.199 & 0.259 & 0.863 & 0.176 & 0.381 & 0.826 \\
\hline \multicolumn{8}{|c|}{ Iranian Cities } \\
\hline \multirow{5}{*}{439} & & \multicolumn{3}{|c|}{$\mathrm{En} \rightarrow \mathrm{Fa}$} & \multicolumn{3}{|c|}{$\mathrm{Fa} \rightarrow \mathrm{En}$} \\
\hline & $1 \mathrm{x} 1$ & 0.196 & 0.334 & 0.846 & 0.109 & 0.280 & 0.840 \\
\hline & $2 \times 2$ & 0.435 & 0.155 & 0.918 & 0.228 & 0.205 & 0.889 \\
\hline & Baseline & 0.175 & 0.353 & 0.840 & 0.057 & 0.282 & 0.842 \\
\hline & DirecTL+ & 0.132 & 0.391 & 0.828 & 0.289 & 0.185 & 0.901 \\
\hline \multirow{5}{*}{469} & & \multicolumn{3}{|c|}{$\mathrm{Ru} \rightarrow \mathrm{Fa}$} & \multicolumn{3}{|c|}{$\mathrm{Fa} \rightarrow \mathrm{Ru}$} \\
\hline & $1 \mathrm{x} 1$ & 0.382 & 0.197 & 0.898 & 0.134 & 0.282 & 0.842 \\
\hline & $2 \times 2$ & 0.525 & 0.139 & 0.926 & 0.252 & 0.237 & 0.870 \\
\hline & Baseline & 0.267 & 0.277 & 0.864 & 0.092 & 0.296 & 0.833 \\
\hline & DirecTL+ & 0.151 & 0.332 & 0.846 & 0.222 & 0.210 & 0.886 \\
\hline \multicolumn{8}{|c|}{ Iranian locations } \\
\hline \multirow{5}{*}{1893} & & \multicolumn{3}{|c|}{$\mathrm{Ru} \rightarrow \mathrm{Fa}$} & \multicolumn{3}{|c|}{$\mathrm{Fa} \rightarrow \mathrm{Ru}$} \\
\hline & $1 \mathrm{x} 1$ & 0.380 & 0.201 & 0.898 & 0.135 & 0.274 & 0.848 \\
\hline & $2 \times 2$ & 0.553 & 0.134 & 0.931 & 0.278 & 0.217 & 0.883 \\
\hline & Baseline & 0.285 & 0.270 & 0.867 & 0.078 & 0.318 & 0.822 \\
\hline & DirecTL+ & 0.155 & 0.345 & 0.847 & 0.317 & 0.189 & 0.900 \\
\hline \multicolumn{5}{|c|}{ Results averaged over all datasets } & & & \\
\hline & $1 \mathrm{x} 1$ & 0.235 & 0.259 & 0.859 & & & \\
\hline & $2 \times 2$ & 0.442 & 0.162 & 0.912 & & & \\
\hline & Baseline & 0.245 & 0.278 & 0.857 & & & \\
\hline & DirecTL+ & 0.311 & 0.253 & 0.878 & & & \\
\hline
\end{tabular}

Table 3: Transliteration results, continued, including overall averaged scores.

may mean that it is possible to use quite small training sets for transliteration, if the data are highly homogeneous and clean. 


\section{Discussion and Current Work}

To summarize, the main contributions of the presented work are: we provide a new, simple, and manually verified data set for evaluation of transliteration models; we apply models built for etymological alignment to the task of cross-lingual transliteration; we introduce simple extensions for prediction to the alignment models, which yield procedures for transliteration based on the alignment. We attempt to ground this work clearly in the context of other related approaches.

The MDL-based Etymon models, applied to the transliteration task without significant modifications, have achieved results that are comparable with stateof-the-art methods reported in the literature. We have discussed how the nature of data, as well as its homogeneity, impacts performance quality.

Current work includes adapting Etymon's context-sensitive models for transliteration. These models were shown, [19], to achieve substantially lower compression cost and normalized edit distance than the $1 \times 1$ and $2 \times 2$ models. We are implementing the prediction algorithm for these models, which is more complex and requires a target language model. Another complication is that the context models require each symbol to be represented as a vector of phonetic features. Thus, the next step will be an implementation of phonetic representations of the data. We also plan to expand our datasets by including more language pairs, and more complex types of data, including company names.

\section{References}

1. Andrews, N., Eisner, J., Dredze, M.: Name phylogeny: A generative model of string variation. In: Proceeding of the 2012 Joint Conference of Empirical Methods in Natural Language Processing and Computational Natural Language Learning (EMNLP-CoNLL) (2012)

2. Atkinson, M., Piskorski, J., van der Goot, E., Yangarber, R.: Multilingual realtime event extraction for border security intelligence gathering. In: Wiil, U.K. (ed.) Counterterrorism and Open Source Intelligence. Springer Lecture Notes in Social Networks, Vol. 2 (2011)

3. Bergsma, S., Kondrak, G.: Alignment-based discriminative string similarity. In: Proceedings of the 45th Annual Meeting of the Association of Computational Linguistics (2007)

4. Ekbal, A., Naskar, S.K., Bandyopadhyay, S.: A modified joint source-channel model for transliteration. In: Proceedings of the COLING/ACL, poster session. Stroudsburg, PA (2006)

5. Finch, A., Sumita, E.: Phrase-based machine transliteration. In: Proceedings of the Workshop on Technologies and Corpora for Asia-Pacific Speech Translation (TCAST) (2008)

6. Goldwasser, D., Roth, D.: Transliteration as constrained optimization. In: Proceedings of the Conference on Empirical Methods in Natural Language Processing (2008)

7. Jiampojamarn, S., Cherry, C., Kondrak, G.: Joint processing and discriminative training for letter-to-phoneme conversion. In: Proceedings of ACL-08: HLT. Columbus, Ohio (2008) 
8. Jiampojamarn, S., Kondrak, G., Sherif, T.: Applying many-to-many alignments and hidden markov models to letter-to-phoneme conversion. In: Human Language Technologies 2007: North American Chapter of the Association for Computational Linguistics. Rochester, New York (2007)

9. Karimi, S., Scholer, F., Turpin, A.: Machine transliteration survey. ACM Computing Surveys 43(3) (2011)

10. Karimi, S., Turpin, A., Scholer, F.: Corpus effects on the evaluation of automated transliteration systems. In: Proceedings of the 45th Annual Meeting of the Association for Computational Linguistics (2007)

11. Li, H., Zhang, M., Su, J.: A joint source-channel model for machine transliteration. In: Proceedings of the 42nd Annual Meeting on Association for Computational Linguistics (2004)

12. Lindén, K.: Multilingual modeling of cross-lingual spelling variants. Information Retrieval 9(3) (2006)

13. Pervouchine, V., Li, H., Lin, B.: Transliteration alignment. In: Proceedings of the Joint Conference of the 47th Annual Meeting of the ACL and the 4th International Joint Conference on Natural Language Processing of the AFNLP (2009)

14. Pouliquen, B., Steinberger, R., Ignat, C., Temnikova, I., Widiger, A., Zaghouani, W., Žižka, J.: Multilingual person name recognition and transliteration. CORELA - COgnition, REpresentation, LAnguage (2005)

15. Schafer, C.: Novel probabilistic finite-state transducers for cognate and transliteration modeling. In: 7th Biennial Conference of the Association for Machine Translation in the Americas (AMTA) (2006)

16. Sherif, T., Kondrak, G.: Substring-based transliteration. In: Proceedings of the 45th Annual Meeting of the Association of Computational Linguistics (2007)

17. Wettig, H., Hiltunen, S., Yangarber, R.: MDL-based Models for Alignment of Etymological Data. In: Proceedings of RANLP: the 8th Conference on Recent Advances in Natural Language Processing. Hissar, Bulgaria (2011)

18. Wettig, H., Nouri, J., Reshetnikov, K., Yangarber, R.: Information-theoretic modeling of etymological sound change. In: Approaches to measuring linguistic differences. Mouton de Gruyter (2013)

19. Wettig, H., Reshetnikov, K., Yangarber, R.: Using context and phonetic features in models of etymological sound change. In: Proceedings of EACL Workshop on Visualization of Linguistic Patterns and Uncovering Language History from Multilingual Resources. Avignon, France (2012)

20. Yangarber, R.: Verification of facts across document boundaries. In: Proc. IIIA2006. Helsinki, Finland (2006)

21. Yangarber, R., Best, C., von Etter, P., Fuart, F., Horby, D., Steinberger, R.: Combining information about epidemic threats from multiple sources. In: Proc. RANLP-2007 MMIES Workshop. Borovets, Bulgaria (2007)

22. Zelenko, D.: Combining MDL transliteration training with discriminative modeling. In: Proceedings of the Named Entities Workshop: Shared Task on Transliteration (2009)

23. Zelenko, D., Aone, C.: Discriminative methods for transliteration. In: Proceedings of EMNLP: Conference on Empirical Methods in Natural Language Processing (2006)

24. Zhang, M., Li, H., Kumaran, A., Liu, M.: Report of news 2012 shared task on machine transliteration. In: Proceedings of NEWS 2012 Named Entities Workshop. vol. $12(2012)$ 